\title{
Disrupción auriculoventricular durante una plastia de la válvula mitral y corrección con parche de pericardio y Bioglue ${ }^{\circledR}$
}

\author{
Atrioventricular disruption during a mitral valve repair and correction \\ with pericardium patch and Bioglue ${ }^{\circledR}$
}

Federico Núñez ${ }^{1}$ Catalina Tovar² ${ }^{*}, G$ Germán Franco ${ }^{3}$, Lucía Casanova ${ }^{4}$, Sonia Pachón ${ }^{5}$

\begin{abstract}
Resumen
La disrupción auriculoventricular después de un cambio de la válvula mitral es una complicación rara, sin embargo presenta altos índices de mortalidad. Este es el primer reporte de un caso de ruptura ventricular luego de una plastia mitral en una paciente femenina de 69 años llevada a plastia mitral y tricuspídea. Después de terminar la perfusión cardiopulmonar, se observó ruptura ventricular localizada en el surco auriculoventricular, el desgarro fue reparado externamente con un parche de pericardio y adhesivo quirúrgico biológico. La paciente se recuperó y fue dada de alta sin complicaciones. Se discute acerca de la etiología y el reparo quirúrgico de esta complicación.

Palabras clave: disrupción auriculoventricular, válvula mitral, parche de pericardio.
\end{abstract}

\begin{abstract}
Atrioventricular disruption after mitral valve replacement is an extremely rare but lethal complication. This is the first case of ventricular disruption following mitral valve repair in a 69 year old female who underwent mitral and tricuspid valve repair. After completion of cardiopulmonary bypass a ventricular rupture was observed at the level of the atrioventricular groove. The tear was externally repaired with pericadial patch and biological surgical adhesive. The patient recovered and was discharged without major complications. We discuss the etiology and surgical repair of this complication.

Key words: atrioventricular disruption, mitral valve, pericardium patch.
\end{abstract}

\section{Introducción}

La ruptura del ventrículo izquierdo fue descrita por primera vez en 1967 por Roberts y Morrow como hallazgo en autopsias de dos pacientes con remplazo mitral ${ }^{(1)}$. Es una compli- cación poco frecuente, está reportada del $0.52 \%$ al $14.3 \%$ de las cirugías de la válvula mitral, con una mortalidad que va del $65 \%$ al $100 \%$ de los casos $^{(2)}$. La alta mortalidad se debe a la localización anatómica de la ruptura por la pobre exposición y a la relación anatómica con la arteria circunfleja. ${ }^{(3)}$

1 Cirujano cardiovascular, Fundación Favaloro-Universidad del Salvador. Buenos Aires, Argentina. Jefe cirugía cardiovascular, Clínica Shaio.

2 Anestesióloga, Universidad Surcolombiana. Fellow de Anestesia Cardiotoracica, Universidad del Rosario.

3 Anestesiólogo cardiovascular, Universidad del Rosario. Anestesiólogo Cardiovascular, Fundación Cardioinfantil.

4 Cirujano cardiovascular, Universidad Javeriana. Rotante institucional cirugía cardiaca congénita en Fundación Cardioinfantil.

5 Enfermera. Especialista en sistemas de garantía de calidad y auditoria de servicios de salud, Fundación escuela de ciencias de la salud. Coordinadora de cirugía cardiovascular, Clínica Shaio.

Correspondencia: Catalina Tovar. Correo electrónico: catalinatovarn@hotmail.com

Recepción: 16/05/2016 - Revisado: 19/09/2016 - Aceptación: 21/11/2016 
Esta requiere un abordaje quirúrgico específico que no está estandarizado.

Se describe a continuación el manejo satisfactorio de una disrupción auriculoventricular después de una plastia mitral en un tejido miocárdico friable que fue reparada de manera externa por aplicación de parche de pericardio y sellante biológico (BioGlue ${ }^{\circledR}$ ), fuera de circulación extracorpórea con resultados favorables.

\section{Caso Clínico}

Paciente de sexo femenino de 69 años, con antecedente de hipotiroidismo, presentó cuadro clínico de deterioro de la clase funcional, disnea y edema de miembros inferiores. El ecocardiograma transtorácico evidenció insuficiencia mitral severa, insuficiencia tricuspídea moderada, con fracción de eyección del ventrículo izquierdo de 55\%; cateterismo cardiaco con arterias coronarias sanas. Fue llevada a cirugía luego de compensación clínica, se realiza esternotomía y en circulación extracorpórea a normotermia y protección miocárdica, se realiza atriotomía derecha y plastia tricuspídea mediante bicuspidización; por abordaje transeptal de aurícula izquierda se visualiza válvula mitral y como hallazgo principal se encuentra ruptura de cuerdas de primer y segundo orden en el festón A3 y extrema friabilidad de los tejidos. Se realiza comisuroplastia y anuloplastia con anillo n. 26, comprobando por prueba hidrostatica adecuada continencia de la válvula. Se cierra septum interventricular y aurícula derecha, sale a ritmo de bloqueo AV, cuando empieza a latir el corazón se observa con cada latido sangrado masivo proveniente de la pared posterior compatible con disrupción auriculoventricular, se explora encontrando desgarro del miocardio en la unión auriculoventricular posterolateral de $3 \mathrm{~cm}$. Por la extrema friabilidad de los tejidos se decide corrección externa de la disrupción con parche de pericardio autólogo 3 x $5 \mathrm{~cm}$ colocado sobre la pared posterolateral, en el sitio de la disrupción y aplicación de $10 \mathrm{cc}$ de BioGlue ${ }^{\circledR}$ puestos en toda la zona de la unión auriculoventricular posterior y lateral del corazón logrando control del sitio de la disrupción (figura 1). Salida de perfusión cardioulmonar con marcapaso por bloqueo AV de III grado, y con infusión de Noradrenalina a 0.05 microgramos por kilo por minuto, tiempo de perfusión fue de 120 minutos, tiempo de isquemia miocardiaca de 69 minutos. Ecocardiograma trasesofágico evidencia fracción de eyección del ventrículo izquierdo conservada del $55 \%$, anillo protésico mitral normofuncionante. Se realiza cierre de esternotomía, y se traslada a la unidad de cuidado intensivo donde permanece por dos días, evolucionando favorablemente y luego de 9 días en hospitalización general se dio egreso.

\section{Discusión}

La disrupción ventricular luego de remplazo de la válvula mitral es una complicación inusual, pero con una elevada mortalidad. La incidencia reportada entre $0.52 \%$ y el $14.3 \%$ de los pacientes que van a remplazo de la válvula mitral con una mortalidad que varía entre el $65 \%$ al $100 \%{ }^{(2)}$, existen reportes de ruptura ventricular luego de remplazo de válvula aortica ${ }^{(4)}$, posterior a revascularización miocárdica ${ }^{(5)}$ pero no encontramos reportes de ruptura después de una plastia mitral, como fue el caso de esta paciente que presentamos.

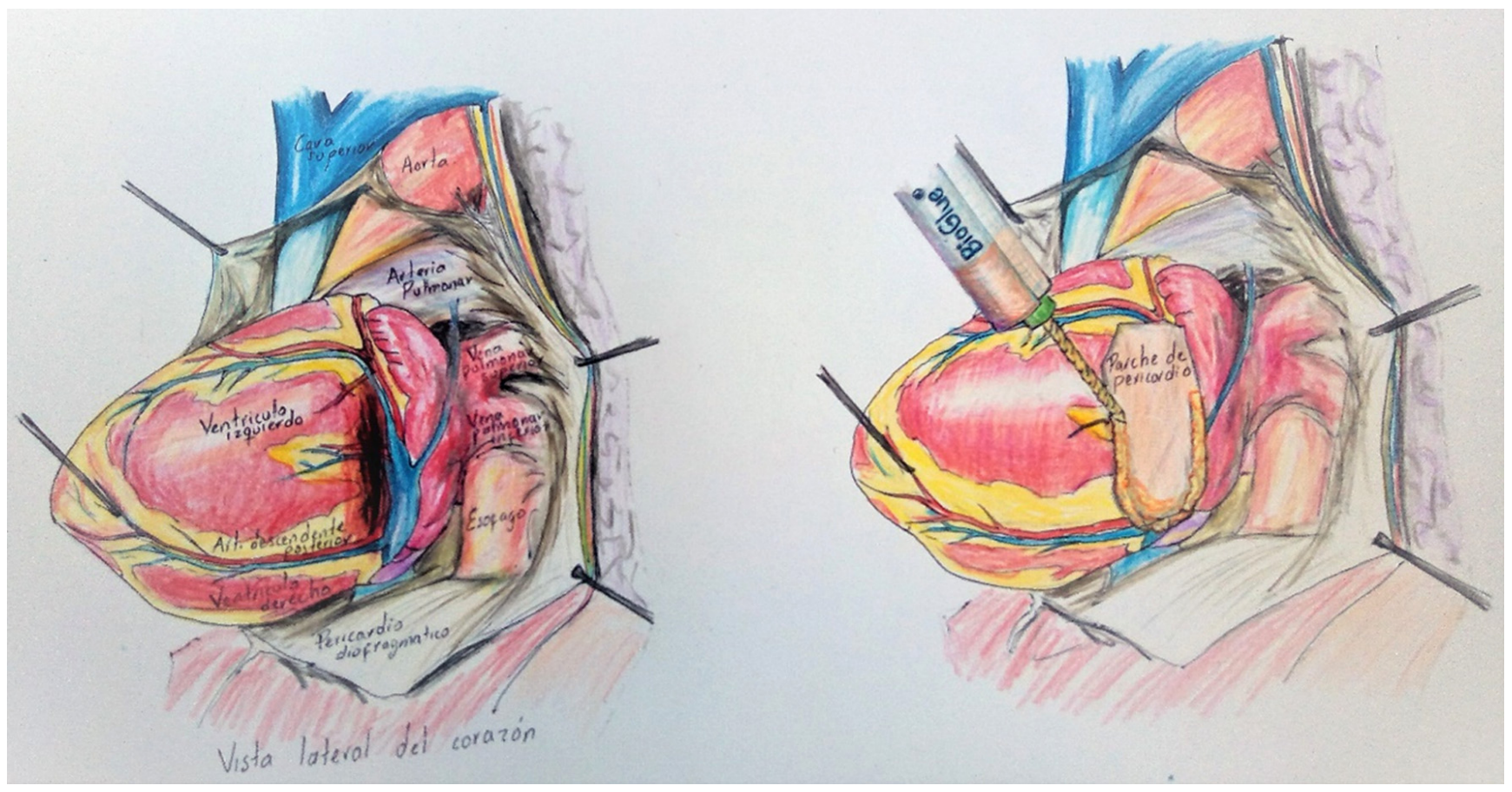

Figura 1. Técnica de reparo con parche pericardio autólogo y BioGlue ${ }^{\circledR}$. 
R.F.S Revista Facultad de Salud

Julio - Diciembre de 2016;8(2):30-33
Disrupción auriculoventricular durante una plastia de la válvula mitral y corrección con parche de pericardio y Bioglue ${ }^{\circledR}$. Reporte de Caso
La elevada mortalidad se debe a las dificultades para la visualización anatómica y determinación del sitio preciso de la ruptura por la pobre exposición, por la friabilidad del musculo que en el momento del reparo no puede sostener bien la sutura y por la localización usual de la disrupción cerca del surco auriculoventricular por donde pasa la arteria circunfleja (figura 2), que puede comprometerse tanto en la ruptura como en el reparo con la subsecuente isquemia e infarto del miocardio ${ }^{(6)}$.

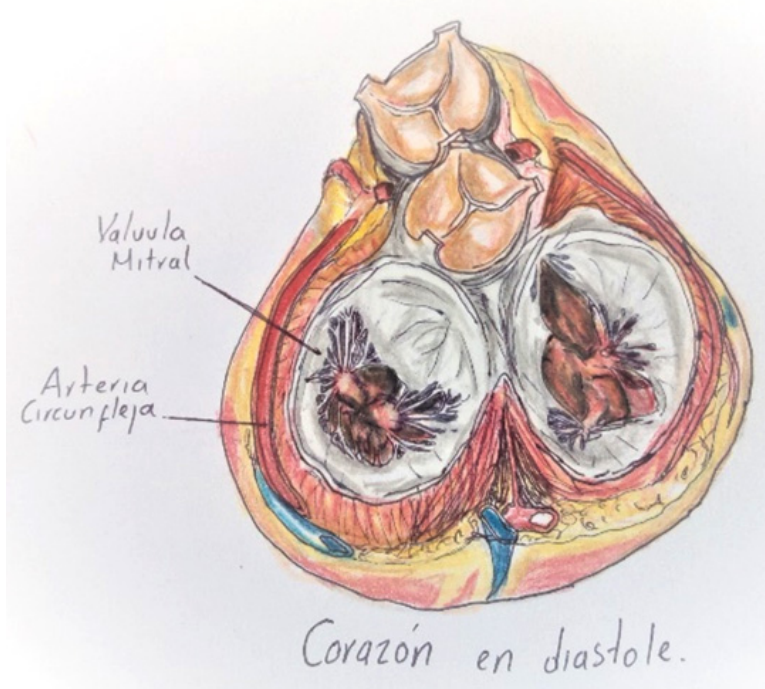

Figura 2. Relación de la válvula mitral con arteria circunfleja.

Los factores de riesgo, implicados en la presentación de la ruptura ventricular relacionados con cirugía de la válvula mitral, son la edad avanzada, enfermedad reumática del corazón, excesiva calcificación del aparato de la válvula mitral, sexo femenino, isquemia miocárdica, endocarditis infecciosa con absceso del anillo, enfermedad miocárdica, hemodiálisis y ventrículo pequeño (diámetro de fin de diástole menor de $50 \mathrm{~mm})$.

Los factores de riesgo intraoperatorio que pueden precipitar la ruptura son causas mecánicas, como la excesiva resección de las valvas mitrales, el anillo y/o el músculo papilar; adherencias de una operación previa, prótesis de gran tamaño, suturas profundas en el miocardio, retracción forzada en el anillo mitral y del ventrículo izquierdo ${ }^{(1)}$. Esta paciente, de edad avanzada, tenía como factor predisponente más importante el miocardio muy friable para que se presentara la ruptura. De cualquier manera la prevención es el factor más importante teniendo especial cuidado en pacientes de riesgo, para reducir la presentación de esta complicación $^{(3)}$. Las precauciones intraoperatorias descritas para minimizar el riesgo cambio valvular mitral son la preservación de la valva posterior, evitar desbridamiento excesivo del anillo calcificado, colocación de tamaño adecuado de la válvula y evitar la tracción excesiva del ventrículo ${ }^{(1)}$. EL ecocardiograma transesofágico intraoperatorio juega un rol importante en la identificación de los pacientes en riesgo y en establecer el diagnóstico temprano seguido de una inmediata intervención quirúrgica. ${ }^{(1)},(7)$

Muchas técnicas de reparo han sido descritas para reparar la ruptura del ventrículo izquierdo incluyendo el uso de la técnica de sándwich ${ }^{(8)}$, técnica de translocación parcial ${ }^{(9)}$, pericardio bovino y sutura continua ${ }^{(3),(10)}$, pericardio bovino y otro tipo de sellante diferente al usado en nuestro caso y soportado con balón de contra pulsación ${ }^{(11)}$, pero de manera general, estas técnicas se pueden clasificar en abordaje interno y abordaje externo. El abordaje interno incluye el cierre directo de la lesión con suturas reforzadas o con parche que cubre la lesión fijado al endocardio intacto del ventrículo y la aurícula a través del anillo, luego de haber retirado la prótesis valvular ${ }^{(6)}$. A pesar de que se ha considerado el reparo interno o endoventricular como el método que mejora la sobrevida ${ }^{(2),(10)}$, encontramos estudios que muestran una sobrevida del 67 al $100 \%$ en procedimientos con reparo externo y $27 \%$ de sobrevida en reparo interno ${ }^{(6)}$, (12), con un número limitado de pacientes en estos estudios; sin embargo, la técnica de reparo externo que fue usada de manera exitosa en esta paciente en quien el reparo externo estaba limitado por la localización posterior y la friabilidad del tejido, representa una técnica de reparo alternativa y efectiva con resultados alentadores ${ }^{(13)}$.

Tradicionalmente el reparo de las heridas quirúrgicas y traumáticas se ha realizado con material de sutura, pero presenta algunos inconvenientes en este tipo de lesiones, como la tensión sobre un tejido miocárdico friable y el riesgo de ligar la arteria circunfleja. Los sellantes tisulares pueden sustituir las suturas convencionales o complementarlas evitando estos riesgos. El Bioglue ${ }^{\circledR}$ es un adhesivo quirúrgico desarrollado para conseguir hemostasia durante procedimientos quirúrgicos y está aprobado por la Food and Drug Administration para su uso desde el año 2000, está compuesto por $45 \%$ de la albúmina sérica bovina purificada y $10 \%$ por glutaraldehído. Cuando se aplica a los tejidos cardiovasculares, las moléculas de glutaraldehído se unen de manera covalentemente a la albumina sérica bovina y a las proteínas tisulares en el sitio de reparación, creando un sello mecánico independiente de la cascada de coagulación $^{(14)}$. En este caso se resolvió de manera exitosa la disrupción auriculoventricular con el uso de este sellante y el parche de pericardio puesto externamente sobre la lesión.

\section{Conclusión}

La disrupción auriculoventricular, luego de procedimientos de la válvula mitral, es una complicación infrecuente pero con alta mortalidad. Se debe tener especial cuidado con los pacientes que tienen factores de riesgo para prevenir esta complicación y, una vez presentada, necesariamente se debe hacer un diagnóstico temprano y el reparo completo de acuerdo con la experiencia del cirujano para tener un mejor resultado, ya que no existe un consenso acerca del manejo, y cada 
vez, se publican más reportes acerca de las técnicas de reparo con buenos resultados. El presente reporte de manejo de esta complicación representa una técnica de reparo alternativa, que en este caso tuvo resultados favorables.

\section{Referencias}

1. Bisoyi S, Mohanty J, Mohapatra R, Nayak D. Left ventricular rupture postmitral valve replacement: surviving a catastrophe. Ann Card Anaesth. 2015;18(1):87-90.

2. Zhang $\mathrm{HJ}, \mathrm{Ma} W G, \mathrm{Xu} J \mathrm{P}, \mathrm{Hu} S \mathrm{~S}$, Zhu XD. Left ventricular rupture after mitral valve replacement: a report of 13 cases. Asian Cardiovasc Thorac Ann. $2006 ; 14(1): 26-9$.

3. Kwon J, Jung T, Lee D. The rupture of atrioventricular groove after mitral valve replacement in an elderly patient. J Cardiothorac Surg. 2014;9(1):28.

4. Nakamura T, Izutani H, Shibukawa T, Higuchi T. ARTICLE IN PRESS Aortoventricular disruption after aortic valve replacement: a rare complication. Interact Cardiovasc Thorac Surg. 2010;1 1:447-8.

5. Jennifer S. Lawton, MD, Seema P. Deshpande M, Paul B. Zanaboni, MD, and Ralph J. Damiano, Jr M. Spontaneous Atrioventricular Groove Disruption During Off-Pump Coronary Artery Bypass Grafting. Ann Thorac Surg. 2005; 79 (1): 339-41

6. Sersar SI, Jamjoom AA. Left ventricular rupture post mitral valve replacement. Clin Med Cardiol. 2009;3: $101-13$.

7. Chui J, Roscoe A, Tsang W. Atrioventricular Disruption After Mitral Valve Replacement: The Role of Intraoperative
Transesophageal Echocardiography. 2014;119 (5):1074-7.

8. Myles E. Lee M. Use of a Sandwich Technique. Tex Hear Inst J. 2014; 41 (2):195-7.

9. Yaku H, Shimada Y, Yamada Y, Hayashida K, Fukumoto A, Watanabe $T$, et al. Partial translocation for repair of left ventricular rupture after mitral valve replacement. Ann Thorac Surg. 2004;78(5):1851-3.

10. Guerrero AF, Valero W, Tavera A, Obando CE, Camacho J, Camilo J. Disrupción auriculoventricular posterior a reoperación de reemplazo valvular mitral: reparación y evaluación ecocardiográfica. Rev Colomb Cardiol. 2016;23(4):333.e1-333.e4.

11. Argiriou M, Patris V, Lama N, Argiriou O, Charitos C. Off pump repair of left ventricular rupture following mitral valve replacement: The crucial assistance of the IntraAortic Balloon Pump, case report. Int J Surg Case Rep. 2013;4(1):5-6.

12. Schuetz A, Schulze C, Wildhirt SM. Off-Pump Epicardial Tissue Sealing - A Novel Method for Atrioventricular Disruption. Ann Thorac Surg . 2004;78:569-74.

13. Dobrilovic N, Raman J, Fingleton JG, Maslow A, Singh AK. Long-Term Outcomes of External Repair as a Rescue Operation for Atrioventricular Groove Disruption Ann Thorac Surg. 2017; 103 (2): 491-96.

14. Bhamidipati CM, Coselli JS, LeMaire SA. BioGlue ${ }^{\circledR}$ in 2011 : What Is lits Role in Cardiac Surgery? J Extra Corpor Technol. $2012 ; 44(1)$ : 6-12. 